\title{
Comparación de dos tratamientos a base de progestágenos para la sincronización de celos ovinos
}

\section{Comparisson of two protocols with progesterone insert for synchronization of estrus in ewes}

Fecha de Recepción: 25 de febrero de 2013

Fecha de Aceptación: 16 de octubre de 2013

\author{
Omar Urete Barrera ${ }^{1}$, José Luis Porras Vargas²
}

\section{Resumen}

Objetivo. Determinar la respuesta a dos programas de sincronización de estros en 30 ovejas vacías. Materiales y métodos. Las ovejas fueron seleccionadas por ecografía y distribuidas al azar en tres grupos de 10 ovejas cada uno. Las ovejas de los grupos 1 y 2 recibieron el día 0 un implante auricular con $3 \mathrm{mg}$ de Norgestomet junto con la inyección intramuscular de 1.5 mg de Norgestomet, $2.5 \mathrm{mg}$ de Valerato de Estradiol y una esponja intravaginal con acetato de medroxiprogesterona respectivamente, el grupo 3 fue el grupo control. El día 8 se inyecto D- Cloprostenol y el día 9 se retiro el implante y la esponja, se realizo detección de celos por 4 días y se realizo servicio con monta natural. El diagnostico de preñez se realizo 30 días después del servicio. Resultados. En el grupo 1, 7 ovejas presentaron estro, 5 de ellas dentro de las 48 horas $(50 \%)$ y 2 de ellas dentro de las 72 horas (20), 3 de las 10 ovejas no presentaron estro (30), en este grupo 3 de las 10 ovejas resultaron preñadas (30\%). En el grupo 2, 4 de las 10 ovejas

\begin{abstract}
Objective. In just farm the response of two estrus synchronization programs was evaluated with 30 nonpregnant ewes, with good body condition score, Materials and metods. They were selected by ultrasonography, they were distribuites at random in three different groups of 10 cows each one. The 1 group received on 0 day $2.5 \mathrm{mg}$ of estradiol valerate and $3 \mathrm{mg}$ of Norgestomet along with an implant of $3 \mathrm{mg}$ s.c. of Norgestomet, in the 2 group the ewes were subjected to estrus synchronization protocols with intravaginal sponges impregnated with medroxiprogesterone acetate (MPA), the 3 group were control group, in both groups 1 and two administered inyection on 8 day D-Cloprostenol, on 9 day the implant with norgestomet and intravaginal sponges were removed each groups respectively, estrus detection during 4 days, the service were by natural breeding. Pregnancy diagnostic were determinated by transrectal ultrasonograafy 30 days after being natural bredding. Results. Of the
\end{abstract}

1 Médico Veterinario y Zootecnista. Profesional IZAGAN. e-mail omarveterinario@yahoo.es

2 Médico Veterinario y Zootecnista, Esp MSc. Docente Medicina Veterinaria y Zootecnia. Universidad Pedagógica y Tecnológica de Colombia, Tunja. e-mail joseluisporrasv@hotmail.com 
presentaron celo, 2 dentro de las 48 horas $(20 \%$ y 2 dentro de las 72 horas (20), 6 ovejas no presentaron estro $(60 \%)$, en este grupo ninguna oveja resulto preñada. En el grupo 3, 1 de las 10 ovejas presento celo dentro de las 24 horas $(10 \%)$, en este grupo 1 oveja resulto preñada $(10 \%)$. Conclusión. El implante auricular con Norgestomet fue el mejor sincronizador de celos ovinos.

Palabras clave: Celos, ovulación, mejoramiento genético (Fuente: DeCS). first protocol were 7 of 10 ewes estrus reponse, 5 inside 48 hours (50\%) and 2 inside 72 hours (20), 3 of 10 ewes does not estrus response $(30 \%)$, in this groups 3 of 10 ewes pregnancy result $(30 \%)$. In the second group 4 of 10 ewes estrus response, 2 inside 48 hours $(20 \%)$ and 2 inside 72 hours $(20 \%), 6$ ewes does nos estrus reponse $(60 \%)$, in this group none ewe pregnancy result. In the three group, 1 of 10 ewe estrus responses inside 24 hours $(10 \%)$, in this group 1 ewe pregnancy result. Conclusion. To sum up that protocol implant with norgestomet were more efficient in synchronizing estrus in ewes.

Key Words: Estrus, ovulation, genetic improvement (Source: DeCS). 


\section{Introducción}

La producción ovina es una fuente importante de proteína de origen animal que posee numerosas ventajas que la sitúan en un lugar preponderante en la producción pecuaria además de ser una explotación generadora de ingresos (1). En la actualidad se observa, en la mayoría de granjas ovinas del país, un manejo reproductivo caracterizado por el uso de reproductores criollos quedifícilmentemejoran los parámetrosproductivos con la genética que poseen, adicionalmente no se valoran las pérdidas por los intervalos entre partos tan largos, que son consecuencia de una serie de factores nutricionales, sanitarios, ambientales y de manejo. Estos aspectos hacen que sea insuficiente la producción para abastecer la demanda de los mercados (2).

Una producción eficiente de carne, leche, lana y pie de cría depende de la eficiencia reproductiva de las hembras, es por ello que la investigación se enfoca a mejorarla con programas de sincronización de estros con progestágenos, metodología que brinda la posibilidad de aplicar la inseminación artificial o la transferencia de embriones que contribuyen al desarrollo genético, zootecnico y sanitario, logrando un impacto positivo en la producción ovina (3).

Para realizar la sincronización se han utilizado los progestágenos, se han efectuado diferentes protocolos modificando dosis, tiempos y vías de administración, sin embargo aun no se ha determinado cual presenta mayor eficiencia con relación a los índices de concepción y preñez, manifestación de celos así como la presentación de reacciones adversas. En el presente estudio se determino la eficacia de dos tratamientos a base de progestágenos realizando la evaluación de los parámetros nombrados anteriormente.

\section{Materiales y Métodos}

Sitio de estudio. El estudio fue desarrollado en la Granja Santa Catalina ubicada en el municipio de Pesca (Boyacá), se extiende aproximadamente entre los $5^{\circ}, 33^{\prime}, 37^{\prime \prime}$ de latitud norte. La precipitación fluvial es de $850 \mathrm{~mm}$ al año, una temperatura media de $13.5^{\circ} \mathrm{C}$ (4). La granja cuenta con un área de pastoreo localizada a $2.631 \mathrm{msnm}$, cuenta con construcciones para dar un manejo de semiestabulación a los animales.

Animales de estudio. Se utilizaron 30 ovejas criollas en periodo seco, entre 2 a 5 años de edad, con condición corporal de 3 a 3.5, peso corporal de 32 a $35 \mathrm{Kg}$, en buen estado de salud según examen semiológico y se utilizaron 4 machos de razas Hampshire y Cheviot adultos que sirvieron como reproductores. Las ovejas se dividieron en dos grupos diferentes completamente al azar, los tres grupos fueron manejados de manera similar, estuvieron en un sistema de semiestabulacion, consumiendo pasto tipo Penissetum clandestidium en horas de la mañana y en horas de la tarde entraban al establo a consumir un suplemento consistente en harina de alfalfa y melaza, además de sal mineralizada al 6\%. Los machos se mantuvieron aislados para controlar los servicios y realizar adecuadamente las observaciones.

Metodología. Las 30 hembras se seleccionaron mediante ultrasonografía transrectal, descartando hembras preñadas o con algún problema reproductivo, una vez conformados los tres grupos se registraron los números de las orejeras para su identificación, se realizaron los tratamientos para cada grupo de la siguiente forma: En el grupo 1 se utilizo un implante auricular con $3 \mathrm{mg}$ de Norgestomet junto con la inyección intramuscular de la porción inyectable que contiene $1.5 \mathrm{mg}$ de Norgestomet y $2.5 \mathrm{mg}$ de Valerato de Estradiol. En el grupo 2 se utilizo una esponja intravaginal con acetato de medroxiprogesterona mas espiramicina para controlar la presentación de vaginitis. En el grupo 3 o grupo control se administro como placebo lactato de ringer vía intramuscular.

La observaciones se realizaron desde el día en el cual se colocaron el implante auricular y la esponja intravaginal respectivamente, para descartar la presentación de reacciones adversas, el día 8 de tratamiento se administro un análogo sintético de prostaglandina F2 alfa (D-Cloprostenol) a los grupos 1 y 2 , el día 9 se retiro el implante auricular.

Los servicios se llevaron a cabo con monta natural a corral, los cuales se llevaron a cabo en tres grupos separados para permitir una mejor observación y los carneros se intercambiaron entre los grupos a fin de minimizar cualquier efecto del 
carnero sobre los resultados a evaluar. Las ovejas fueron expuestas a los carneros a las 24 horas de retiradas las esponjas y el implante auricular respectivamente, se realizo la observación de detección de celos cada 12 horas durante 3 horas durante 4 días, se registraron las que mostraban celo y fueron servidas retirándolas del grupo. Las que no mostraron celo durante este lapso de tiempo continuaron con servicio a campo. El diagnostico de preñez se realizo 30 días después de los servicios mediante ecografía.

Durante el estudio se registró la presentación de celos, momento de la presentación de celos en cada grupo y el número de ovejas preñadas.

Diseño estadístico. El diseño estadístico manejado para esta investigación fue descriptivo con variables de tipo cuantitativo, se determino la diferencia porcentual entre los diferentes tratamientos en cuanto a la presentación de celos, tasa de preñez y tasa de concepción. Las diferencias mínimas entre los grupos fueron inferidas de la comparación de los porcentajes entre grupos y por medio de la comparación entre grupos.

\section{Resultados y Discusión}

Presentación de celos. En el grupo experimental 1; de las diez ovejas tratadas, presentaron celo siete ovejas que corresponde al $70 \%$, de las cuales $50 \%$ entra en celo a las 48 horas y el $20 \%$ restante a las 72 horas de retirado el implante auricular. El $30 \%$ de los animales de este grupo no presentaron conducta estral.

Este resultado se encuentra en concordancia con el obtenido por Mendoza et al (2000) quien muestra un $80 \%$ de efectividad del norgestomet como inductor de celo en ovinos en un intervalo entre 24 y 72 horas después de retirado el implante. El norgestomet si bien esta indicado en medicina veterinaria para bovinos, se valoró su efecto en ovinos usándolo a mitad de la dosis, y se observó que indujo la conducta estral en la mayoría de los animales tratados, independientemente de la fase del ciclo en que se encontraban.

La progesterona y el estradiol se han utilizado para bloquear la secreción de LHyFSH, respectivamente, con relativo éxito. Sin embargo, nuevamente, la efectividad de ambos tratamientos depende del estado de desarrollo folicular. Los esteroides que se administran antes o inmediatamente después del inicio de la oleada folicular, corresponden con un retardo en la selección y con un folículo de menor tamaño. Cuando estos se aplican en animales que tienen un folículo dominante establecido, causan atresia del mismo, dependiendo de la dosis, puede llegar a retrasar el reclutamiento de la nueva oleada, actualmente se usa progesterona y estrógenos en conjunto, con lo cual se induce el inicio de una nueva onda folicular entre 3 y 5 días después de retirado el implante, mejorando el grado de sincronización de los animales.

En el grupo 2 en el cual se utilizo la esponja intravaginal impregnada con acetato de medroxiprogesterona (MAP) condujo a que 4 ovejas mostraran celo que corresponde al $40 \%$, de las cuales la mitad tuvo celo a las 48 horas, y la otra mitad a las 72 horas de retirada la esponja intravaginal, seis ovejas (60\%) de los diez animales tratados no presentaron celo.

Comparando este resultado con el obtenido por Viñoles (2001) quien reporta $100 \%$ y $69 \%$ de efectividad en la presentación de celos mediante tratamiento con esponja intravaginal impregnada con acetato de medroxiprogesterona por 12 y 6 días respectivamente, y a los de Ewes (2002) con $80 \%$ en presentación de celos al usar acetato de medroxiprogesterona por seis días y prostaglandina al retiro de las esponjas, se presume que la duración de los tratamientos influye directamente en la presentación de celos.

Buratovich (2000) señala que la duración de los tratamientos con progestágenos influye en el porcentaje de ovejas en celo así como en la fertilidad obtenida, los tratamientos largos demuestran una efectiva capacidad en inducir celos en menor tiempo, pero una relativa baja fertilidad, y tratamientos cortos producen una mayor dispersión de los celos pero la fertilidad es mejor, si las ondas foliculares emergen cada cuatro a seis días, no parece justificado el uso de tratamientos hormonales tan prolongados, la menor fertilidad con tratamientos largos se encuentra asociada con la ovulación de folículos con vida media prolongada (6) lo que sustenta la hipótesis que los tratamientos tradicionales (10- 12 
días) promueven la ovulación de ovocitos "viejos" que tienen poca capacidad para ser fertilizados o si ella ocurre el desarrollo embrionario es anormal resultando en muerte embrionaria prematura.

Knigths et al (2001) reporta resultados similares a los de los anteriores estudios, sincronizo ovejas con progesterona intravaginal y hormona folículo estimulante ( $\mathrm{FSH}$ ) por nueve días, obteniendo $79 \%$ de efectividad en presentación de celos y $40 \%$ de preñez y a los de Catalano et al (2005) al tratar 55 ovejas lactando y 45 ovejas en periodo seco con acetato de medroxiprogesterona en esponja intravaginal, obtuvo de las ovejas lactando el $76.4 \%$ y de las ovejas en periodo seco el $88.9 \%$ en presentación de celo, y $54.0 \%$ y $68.3 \%$ de preñez respectivamente.

Consecuentemente se observa una gran diferencia de los porcentajes de presentación de celos reportados por los anteriores estudios con el $40 \%$ obtenido para la esponja intravaginal en esta investigación. Es posible que la elaboración artesanal de la esponja, pudiera influir en estos bajos resultados, respecto de los obtenidos por los autores señalados anteriormente, esto pudo ser debido a la inadecuada distribución uniforme del producto activo en la esponja que hace que no se alcancen las concentraciones sanguíneas necesarias del progestágeno para sincronizar la dinámica folicular, ya que se ha demostrado que la concentración de progesterona circulante juega un rol preponderante en el recambio folicular en los pequeños rumiantes y que los niveles sanguíneos de progesterona afectan directamente la dinámica folicular y la fertilidad (2).

En cuanto a la duración del tratamiento en esta investigación, se puede concluir que es adecuada, ya que la administración del progestageno no excedió la duración de la vida media fisiológica del cuerpo lúteo (11 a 12 días), permitiendo la ovulación de ovocitos en condiciones óptimas para la fertilización, razón por la cual no se le atribuye a esta situación el bajo porcentaje de presentación de celos y de fertilidad obtenidos.

De conformidad con lo descrito en la metodología sobre la condición corporal de los animales de experimentación, fue adecuada en términos de edad, peso corporal y estado de salud, lo cual descarta la posibilidad de que estas condiciones pudieran influir en la presentación de celos o en la fertilidad, lo relevante durante la investigación fue la disponibilidad limitada del forraje por la temporada de verano.

Se concluye que definitivamente el factor mas incidente en la baja respuesta al protocolo de sincronización conformado por la esponja intravaginal impregnada con acetato de medroxiprogesterona fue la elaboración misma de la esponja, que no permitió la adecuada distribución del principio activo y por ende no hubo una buena absorción para lograr niveles sanguíneos apropiados de progesterona que asegurara el control de la dinámica folicular.

El grupo control presentó una oveja en celo durante las primeras 24 horas de la fase de detección, nueve ovejas del total de este grupo no presentaron celo, esto es un alto porcentaje $(90 \%)$ de animales que se encuentran en anestro permanente durante esta época del año, y que inciden razonablemente en la rentabilidad del productor.

Por consiguiente y según este estudio los tratamientos hormonales para la sincronización de celos en los ovinos pueden ser una alternativa para reanudar la actividad ovárica y mejorar la prolificidad en hembras anestricas.

Lo anterior se basa en que al aumentar la concentración circulante de progesterona; paradójicamente la progesterona que durante el ciclo estral actúa disminuyendo la frecuencia de pulsos de $\mathrm{LH}$, durante el anestro se piensa que bloquea la acción inhibitoria del estradiol sobre el hipotálamo resultando en un aumento de los pulsos de LH. Esto trae la maduración final del folículo, aumenta la secreción de estradiol e induce la retroalimentación positiva y el pico preovulatorio de $\mathrm{LH}$ al retirar el progestágeno, por tanto puede ser una alternativa para la inducción de ciclicidad en ovejas anestricas.

Los animales de este grupo a pesar de recibir el mismo manejo en cuanto a nutrición, sanidad, y de encontrarse en las mismas condiciones medio ambientales en la granja, poseen un factor supresor en el eje hipotálamo-hipofisiario-gonadal que no 
les permite reproducirse durante esta temporada del año. Se puede pensar que uno de los factores importantes que condicionaron el anestro en estos animales es la escasa disponibilidad de forraje durante el verano en que se llevo a cabo el estudio.

Otro factor sin evaluar en este estudio y que afecta indirectamente la reproducción en las ovejas del trópico, es el fotoperíodo (horas luz), que inhibe la actividad ovárica por medio de los efectos luminosos que son captados por el sistema nervioso y transmitidos al sistema endocrino que genera hormonas como la melatonína, la cual dependiendo de sus concentraciones plasmáticas, sensibiliza al hipotálamo para la liberación de GNRH y por ende de $\mathrm{LH}$, condicionando el comportamiento reproductivo de las ovejas, fenómeno descrito por Yellon y Olster en 1985, por lo anterior, y teniendo en cuenta que el estudio se realizo en los meses de verano, existe la posibilidad de un efecto negativo sobre la respuesta a tratamientos de sincronización en esta época del año. Sin embargo las técnicas de sincronización de celos pueden ser una valiosa herramienta para que los productores ovinos mejoren sustancialmente sus ingresos.

Comparación entre grupos. Según las diferencias de porcentajes los animales del grupo1 y grupo 2 presentaron diferencia porcentual con respecto al grupo control, que tan solo una ovejas entro en celo $(10 \%)$ a las 24 horas, y nueve de ellas $(90 \%)$ no presentaron celo, mientras que en el grupo1 siete de las ovejas presentaron celo $(70 \%)$, de las cuales cinco entran en celo a las 48 horas, dos a las 72 horas y tan solo tres no presentan celo. Por otra parte el grupo 2 presenta cuatro ovejas en celo que corresponde a $(40 \%)$ de las cuales dos entra en celo a las 48 horas, y dos a las 72 horas, seis ovejas de este grupo no respondieron al tratamiento.

Observando la gráfica 4 se observa que el grupo CRESTAR $^{\circledR}$ fue el mas eficiente en la presentación de celos, al compararlo con los grupos MAP y CONTROL. De los grupos 1 y 2 se puede inferir que el grupo1 presenta mas animales en celo que el grupo 2, por lo tanto se puede indicar que el grupo 1 supera en aproximadamente $30 \%$ al grupo 2 en presentación de celo y en $60 \%$ al grupo control, igualmente se observa que grupo 1 y 2 , mostraron una concentración de celos dentro de las primeras 72 horas de retirados los tratamientos; ninguno de los tres grupos mostró celo después de las 96 horas postratamiento.

Según este estudio resulta mas viable el implante auricular en la inducción y sincronización de celos ovinos al compararlo con la esponja intravaginal impregnada con MAP y el grupo control, confrontando los resultados con lo descrito por Rubianes et al (2001) quien obtuvo con esponja intravaginal (MAP) $85 \%$ y a los de Mendoza et al (2000) con $88 \%$ en presentación de celos usando norgestomet, se observa que la experiencia para este estudio fue inferior a la reportada por estos autores. La hipótesis para el grupo 1 que incluye estrógenos en su protocolo, es que esta

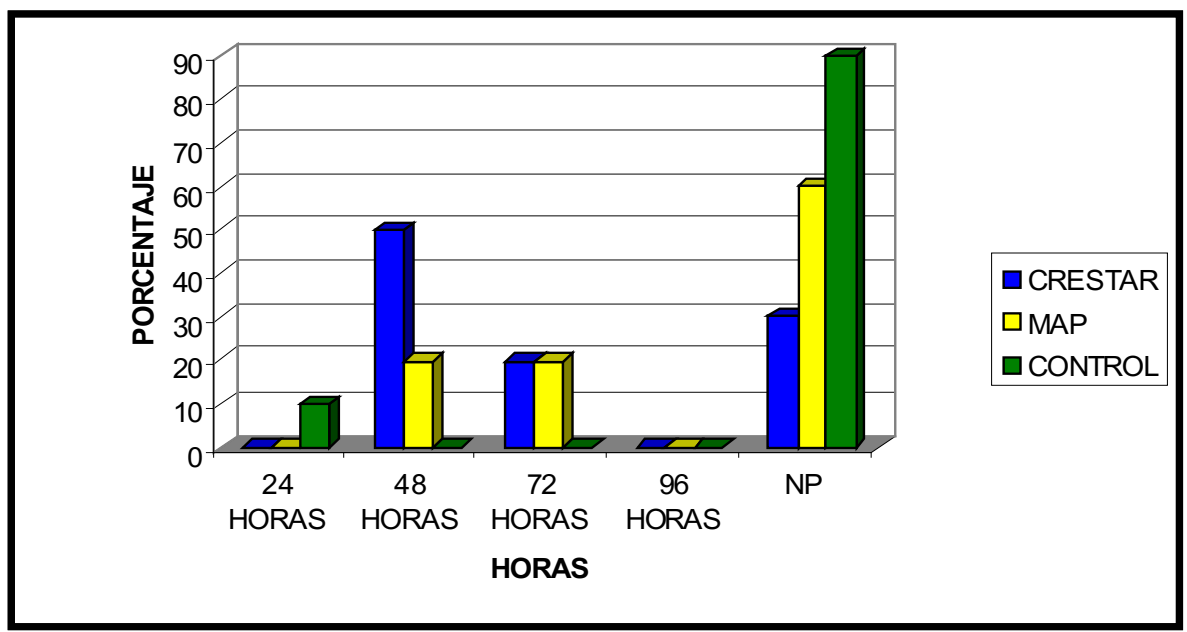

FIGURA1. PRESENTACIÓN DE CELOS (NP) EN LOS TRES TRATAMIENTOS. 
hormona es capaz de producir conducta estral en ovejas, independientemente de la actividad ovárica del animal según lo demuestra Mendoza et al (2000) en su estudio realizado en ovejas ovariectomizadas, lo cual puede sustentar el alto porcentaje de ovejas en celo para este grupo. Para el grupo 2 como se menciono anteriormente pudo influir la elaboración de la esponja sin ninguna estandarización.

Fertilidad. Dado que la investigación pretende además mostrar la fertilidad obtenida para cada uno de los tratamientos de sincronización, se analiza ahora el porcentaje de preñez y de concepción que se obtuvieron para los grupos en estudio.

Como se observa en las gráficas siguientes, los tratamientos a base de progestágenos: implante auricular y esponja intravaginal impregnada con (MAP) si bien mostraron efectividad para inducir el celo, la fertilidad resultante fue solo de un 30 Y $0 \%$ respectivamente, al compararlo con el grupo control que del $10 \%$ que presentó celo todas preñaron, lo anterior esta en concordancia con lo expresado por otros autores $(6,10)$ en cuanto a que la fertilidad de celos inducidos, es en la práctica, menor que la observada en celos naturales. Mendoza et al (2000) menciona que al usar este implante junto al valerato de estradiol, se pueden presentar concentraciones residuales de estrógenos en sangre al retirar el implante, que inducen la conducta estral mas no la ovulación de un folículo por lo cual se observan un porcentaje de fertilidad relativamente bajo para este grupo.
El grupo 2 (MAP) por su parte no mostró ningún porcentaje de preñez para este estudio, se puede inferir que este resultado es consecuencia de la mala distribución del producto activo en la esponja, lo cual conlleva que no se alcancen concentraciones sanguíneas optimas del progestágeno que actúen controlando la dinámica folicular y la secreción de hormonas por parte de la hipófisis, según estudio adelantado por Gibbons et al 2000, reportan una menor fertilidad de los tratamientos con progestágenos en ovejas, debido a una reducción de la migración espermática a nivel del cérvix y acortamiento del período de estro e inhibición de las contracciones uterinas. Lo que podría explicar que de las cuatro ovejas que presentaron celo para el grupo 2 ninguna quedo preñada.

Sin embargo es de señalar que los tratamientos hormonales tienden por favorecer el inicio de la actividad ovárica y de esta forma terminar con el anestro en que se encuentra el animal, reduciendo los días abiertos y ofreciendo ventajas al productor, como se menciono anteriormente. Se puede observar que el grupo que no recibió ningún tratamiento hormonal, tan solo una oveja mostró conducta estral para esta época del año lo cual se traduce en perdidas para el productor.

Analizando los porcentajes de preñez según el tiempo de presentación de celos, se tiene que el grupo 1 presento tres ovejas preñadas, de las cuales dos de las ovejas (20\%) que presentaron celo durante las primeras 48 horas y una que presentó a las 72 horas quedaron preñadas, por

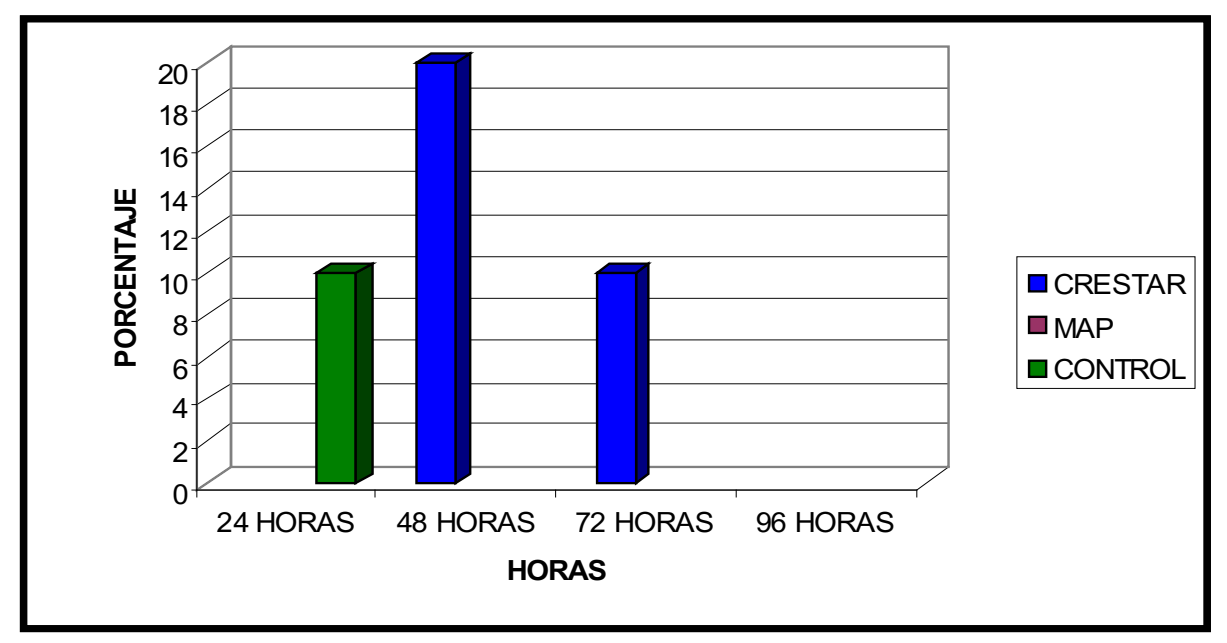

FIGURA 2. PORCENTAJES DE PREÑEZ SEGÚN PRESENTACIÓN DE CELOS. 
su parte el grupo de la esponja intravaginal no presentó ningún animal preñado. Mientras que para el grupo control la oveja que entro en celo en las primeras 24 horas quedó preñada. El tiempo de presentación de celo esta sujeto a la fase del ciclo en que se encuentre el animal. Se determinó que los dos tratamientos sincronizadores, concentraron la presentación de celo en las primeras 72 horas de retirado el tratamiento.

\section{Referencias bibliográficas}

(1) Gibbons AE. Cueto, MI. Transferencia de embriones ovinos y caprinos. Área de investigación en producción animal. Centro regional Patagonia, México; 2000: 47 p.

(2) Rubianes E, Menchaca A. Nuevos tratamientos asociados con la inseminación artificial a tiempo fijo en pequeños rumiantes. Facultad de Veterinaria; Laboratorio de Fisiología de la Reproducción, Universidad de Montevideo, 2000.

(3) Ewes-Pawel M. Effects of a 6-day treatment with medroxyprogesterone acetate after prostaglandin $f_{2}$-induced luteolysis at midcycle on antral follicular development and ovulation rate in nonprolific western whitefacedl. Department of Veterinary Biomedical Sciences, University of Saskatchewan, 2002.

(4) Vargas-De-Castañeda M. Historia de pesca, pueblo grande: dominio de la fortaleza. Editorial J Mar Ltda, 2003: 35-38.
(5) Mendoza M, Ceron J, Ramirez N, Almeraya A. Los tratamientos sincronizadores de estro en combinación con estrógenos, inducen conducta estral en ovejas ovariectomizadas. Facultad de Medicina Veterinaria y Zootecnia, Universidad Autónoma de México, 2000.

(6) Viñoles C. Effect of long-term and shortterm progestagen treatment on follicular development and pregnancy rate in cyclic ewes. Theriogen 2001; 55: 993-1004.

(7) Buratovich OF. Desarrollo de Sistemas Intensivos de Producción de Carne Ovina. Actualización en Producción Ovina. INTA EEA Bariloche; 2000: 34-46.

(8) Knights MT, Hoehn PE, Lewis EK. Effectiveness of intravaginal progesterone inserts and $\mathrm{FSH}$ for inducing synchronized estrus and increasing lambing rate in anestrous ewes. Division of Animal and Veterinary Sciences, West Virginia University, 2001.

(9) Catalano R, González C, Teruel M, Cabodevila J, Callejas S. Efecto del estado fisiológico y del porcentaje de raza Frisona sobre la respuesta reproductiva de ovejas en servicio de primavera. Facultad de Ciencias Veterinarias, Universidad Nacional del Centro de la Provincia de Buenos Aires, 2005.

(10) Rubianes E, Ungerfeld R, De-Castro T. Inducción y sincronización de celos en ovejas y cabras. Tercer Simposio Internacional de Reproducción Animal, Córdoba-Argentina; 2001. 